\begin{tabular}{|c|l|}
\hline Title & Blow-up at space infinity for nonlinear heat equations \\
\hline Author(s) & Giga, Y oshikazu; Seki, Y ukihiro; Umeda, Noriaki \\
\hline Citation & Hokkaido University Preprint Series in Mathematics, 856, 1-17 \\
\hline Issue Date & 2007 \\
\hline DOI & 10.14943/84006 \\
\hline Doc URL & http://hdl.handle.net/2115/69665 \\
\hline Type & bulletin (article) \\
\hline File Information & pre856.pdf \\
\hline
\end{tabular}

Instructions for use 


\title{
Blow-up at space infinity for nonlinear heat equations
}

\author{
Yoshikazu Giga, Yukihiro Seki and Noriaki Umeda \\ Graduate School of Mathematical Sciences, \\ University of Tokyo, \\ 3-8-1 Komaba, Meguro-ku Tokyo 153-8914, Japan
}

\section{Introduction}

This is a survey paper on blow-up phenemena at space infinity for nonlinear heat equations. We are interested in a blow-up problem of the Cauchy problem for nonlinear heat equations

$$
\left\{\begin{array}{l}
u_{t}=\Delta u+f(u), \quad x \in \mathbf{R}^{N}, t>0, \\
u(x, 0)=u_{0}(x), \quad x \in \mathbf{R}^{N} .
\end{array}\right.
$$

Here the nonlinear term $f$ and initial data $u_{0}$ are assumed to satisfy the following conditions;

$f$ is a locally Lipschitz continuous function in $[0, \infty)$ and

$$
\text { fulfills } f(\xi)>0 \text { for } \xi>0 \text { and } \int_{1}^{\infty} \frac{d \xi}{f(\xi)}<\infty
$$

$u_{0}$ is a nonnegative bounded continuous function in $\mathbf{R}^{N}$.

The last condition of $(A 1)$ forces $f$ to grow superlinearly at infinity.

A nonlinear evolution equation may have a unique local-in-time solution in a suitable function space and it can be extend as a solution together with evolution of time so long as it belongs to the function space. However, in general, the Cauchy problem is not solvable globally in time; a solution may blow up in finite time. That is, there may exist a finite time $T<\infty$ such that the solution ceases to exist in the function space at the time $T$. This phenomenon is called blow-up in finite time and we call such a time $T$ blow-up time. The Cauchy problem (1.1) has a unique local-in-time solution $u=u(\cdot, t)$ in $L^{\infty}\left(\mathbf{R}^{N}\right)$ for any nonnegative initial data $u_{0} \in L^{\infty}\left(\mathbf{R}^{N}\right)$. However, it may blow up in finite time. For instance, if the initial data does not decrease at space infinity, the solution of (1.1) does blow up in finite time. We are interested in the blow-up times of solutions and detailed behavior of solutions at the blow-up times. In particular, we discuss solutions which blow up at space infinity as we will state later. 


\section{Various Concepts}

Let $u$ be a blow-up solution to the problem (1.1) with initial data $u_{0}$. The notation $\|\cdot\|_{\infty}$ stands for usual sup norm in $\mathbf{R}^{N}$. By a blow-up time, we mean

$$
\begin{array}{r}
t_{b}\left(u_{0}\right):=\sup \left\{\tau>0 ; u(\cdot, t) \text { is bounded in } \mathbf{R}^{N}\left(\text { i.e. }\|u(t)\|_{\infty}<\infty\right)\right. \\
\text { for } 0<t<\tau\} .
\end{array}
$$

If $t_{b}\left(u_{0}\right)<\infty$, the solution cannot live in $L^{\infty}\left(\mathbf{R}^{N}\right)$ beyond the time $t_{b}\left(u_{0}\right)$. Therefore it does hold that $\|u(t)\|_{\infty} \rightarrow \infty$ as $t \nearrow t_{b}\left(u_{0}\right)$. A point in $\mathbf{R}^{N}$ where $u$ is not locally bounded is called a blow-up point. The blow-up set of $u$ is defined as a set of all blow-up points. Many researchers have tried to characterize blow-up sets. There is a huge literature on this topic. Notice that a blow-up set may possibly be empty even if a finite time blow-up occurs. We are just going to discuss such phenomena. We define blow-up at space infinity as follows:

Definition 2.1. A solution $u$ of (1.1) with blow-up time $t_{b}\left(u_{0}\right)<\infty$ is said to blow up at space infinity if there exists a sequence $\left\{\left(x_{n}, t_{n}\right)\right\} \subset$ $\mathbf{R}^{N} \times\left(0, t_{b}\left(u_{0}\right)\right)$ such that

$$
\left|x_{n}\right| \rightarrow \infty, t_{n} \nearrow t_{b}\left(u_{0}\right) \text { and } u\left(x_{n}, t_{n}\right) \rightarrow \infty .
$$

We define $T_{M}=t_{b}(M)$ with $M=\left\|u_{0}\right\|_{\infty}$, which coincides with the blowup time of the solution $v_{M}(t)$ of the corresponding problem for ordinally differential equation;

$$
\left\{\begin{array}{l}
v^{\prime}=f(v), \quad t>0 \\
v(0)=M
\end{array}\right.
$$

It is easily seen that a unique solution to (2.1) exists and it is expressed as

$$
v_{M}(t)=G^{-1}\left(T_{M}-t\right) \quad \text { with } \quad G(v)=\int_{v}^{\infty} \frac{d \xi}{f(\xi)}
$$

where $G^{-1}$ is the inverse function of $G$. Clearly, $T_{M}=G(M)$. For example, if $f(u)=u^{p},(p>1)$ then $v_{M}(t)=\kappa\left(T_{M}-t\right)^{-1 /(p-1)}$ with $\kappa=(p-1)^{-(p-1)}$ and $T_{M}=(p-1)^{-1} M^{-p+1}$ and if $f(u)=e^{u}$, then $v_{M}(t)=-\log \left(T_{M}-t\right)$ with $T_{M}=e^{-M}$. By the comparison principle, we have $\|u(\cdot, t)\|_{\infty} \leq v_{M}(t)$ for any solution $u$ to the problem (1.1). Thus, in general, $t_{b}\left(u_{0}\right) \geq T_{M}$.

Definition 2.2. Let $u_{0}$ belong to $L^{\infty}\left(\mathbf{R}^{N}\right)$ and set $M=\left\|u_{0}\right\|_{\infty}$. A solution $u$ to the problem (1.1) with initial data $u_{0}$ is said to blow up at minimal blow-up time or the least (possible) blow-up time provided that $t_{b}\left(u_{0}\right)=T_{M}$.

By definition and the strong maximum principle $([12])$, one is able to show 
that a solution with minimal blow-up time necessarily blows up at space infinity:

Theorem 2.3. Assume (A1) and (A2). Suppose that the solution $u$ of (1.1) with initial data $u_{0}$ blows up at minimal blow-up time. Then

$$
\|u(\cdot, t)\|_{\infty}=\lim _{R \rightarrow \infty} \sup _{|x| \geq R} u(x, t)=v_{M}(t) \text { in }\left[0, T_{M}\right) .
$$

Hence, in particular, the initial data $u_{0}$ should satisfy

$$
\lim _{R \rightarrow \infty} \sup _{|x| \geq R} u_{0}(x)=M .
$$

Moreover, the solution $u$ blows up at space infinity. Namely, there exists a sequence $\left\{\left(x_{n}, t_{n}\right)\right\} \subset \boldsymbol{R}^{N} \times\left(0, T_{M}\right)$ such that

$$
\left|x_{n}\right| \rightarrow \infty, t_{n} \nearrow T_{M} \text { and } u\left(x_{n}, t_{n}\right) \rightarrow \infty \text { as } n \rightarrow \infty .
$$

Corollary 2.4. Assume the same hypotheses with Theorem 2.3. Then there exists a "direction" $\psi \in S^{N-1}$, where $S^{N-1}$ is $(N-1)$-dimensional unit sphere, such that

$$
\lim _{k \rightarrow \infty} \frac{x_{n(k)}}{\left|x_{n(k)}\right|}=\psi
$$

for some subsequence $\left\{\left(x_{n(k)}, t_{n(k)}\right)\right\} \subset\left\{\left(x_{n}, t_{n}\right)\right\}$.

The proofs of these results are due to [14]. We give a sketch of the proofs in the next section.

Remark 2.5. Condition (2.3) is not sufficient to raise a blow-up at space infinity. We will introduce some necessary and sufficient conditions on initial data for a solution to blow up at space infinity at minimal blow-up time.

Definition 2.6. Let $u$ be a blow-up solution to the problem (1.1). A "direction" $\psi \in S^{N-1}$ is said to be a blow-up direction of $u$ if there exists a sequence $\left\{\left(x_{n}, t_{n}\right)\right\} \subset \mathbf{R}^{N} \times\left(0, t_{b}\left(u_{0}\right)\right)$ such that

$$
\left|x_{n}\right| \rightarrow \infty, \frac{x_{n}}{\left|x_{n}\right|} \rightarrow \psi, t_{n} \nearrow t_{b}\left(u_{0}\right) \text { and } u\left(x_{n}, t_{n}\right) \rightarrow \infty \quad \text { as } n \rightarrow \infty .
$$

If the solution has at least one blow-up direction, we say that directional blow-up arises. Corollary 2.4 asserts that if the solution of (1.1) blows up at minimal blow-up time, then directional blow-up does occur. If a direction $\eta \in S^{N-1}$ is not a blow-up direction, we call it non-blow-up direction. Our interests are characterizing blow-up set and blow-up directions by behavior of initial data. 
Blow-up problems for nonlinear parabolic equations have been studied for a long time. However, many researchers consider the Cauchy problem only with initial data which decay at space infinity or the Dirichlet problem in a bounded domain. Blow-up at space infinity does not occur for such problems. Only a few researchers studied blow-up at space infinity as far as we know. Let us recall a few results concerning blow-up at space infinity.

The first result in this topic is due to Lacey [10]. He considered the Cauchy-Dirichlet problem in one-dimensional half line;

$$
\left\{\begin{array}{l}
u_{t}=\Delta u+f(u), \quad \text { in }(0, \infty) \times(0, T), \\
u(0, t)=1, \quad t \in(0, T), \\
u(x, 0)=u_{0}(x), \quad \text { in }(0, \infty)
\end{array}\right.
$$

and proved that the solution blows up only at space infinity.

Giga and Umeda [6] considered the Cauchy problem for equation (1.1) with $f(u)=u^{p}(p>1)$ in higher dimension. Assuming $\lim _{|x| \rightarrow \infty} u_{0}(x)=$ $\left\|u_{0}\right\|_{\infty}$ and $u_{0} \not \equiv\left\|u_{0}\right\|_{\infty}$, they proved that the solution blows up at minimal blow-up time and blow-up occurs only at space infinity. They $([7,8])$ dealt with the Cauchy problem (1.1) with general nonlinear term $f$ satisfying condition (GU). They weakened condition on initial data, which leads to a refinement sufficient condition for blow-up at space infinity and discussed directional blow-up. They characterized blow-up directions according to the behavior of the mean value of $u_{0}$ on ball. Although they also deals with sign-changing solutions, we discuss only nonnegative solutions for simplicity.

Seki, Suzuki and Umeda [14] not only generalized the results of [7] to degenerate quasilinear parabolic equations $u_{t}=\Delta \phi(u)+f(u)$ but also gave necessary and sufficient conditions for a solution to blow up at minimal blow-up time and conditions for a direction to be a blow-up direction. The equation is a generalization of porus medium equation with reaction term; $u_{t}=\Delta u^{m}+f(u)$ with $m \geq 1$. Moreover, the nonlinear term $f$ can be taken from very wide class of functions. For example, $f(u)=(1+u)\{\log (1+u)\}^{\beta}$ with $\beta>2$ is allowed in [14]. One of the author obtained the same results for a quasilinear parabolic equation which is a generalization of fast diffusion equation $u_{t}=\Delta u^{m}+f(u)$ with $0<m<1$ in [13], although the assumption of $f$ is a little stronger than [14].

This note is organized as follows. We introduce typical results in the next section and show main ideas of their proofs in $\S 4$. In the final section we show, as applications of their results, some examples of directional blowup, such as a solution which has a single blow-up direction and a solution whose set of blow-up directions coincides with arbitrary given closed set in $S^{N-1}$. We also prove the solution does not blow up at minimal blow-up time if the initial data is an almost periodic function. 


\section{Typical results}

We set $\rho(x)=\left(\int_{\mathbf{R}^{N}} e^{-|y|} d y\right)^{-1} e^{-|x|}$. The mean value function of $u_{0}$ with weight $\rho$ is defined by

$$
A_{\rho}\left(x ; u_{0}\right)=\int_{\mathbf{R}^{N}} \rho(y-x) u_{0}(y) d y,
$$

which plays significant roles. For a direction $\psi \in \mathbf{R}^{N}$, we consider some conditions on $u_{0}$ for $\psi$ to be a blow-up direction.

There exists a sequence $\left\{x_{n}\right\} \subset \boldsymbol{R}^{N}$ such that

$$
\left|x_{n}\right| \rightarrow \infty, \frac{x_{n}}{\left|x_{n}\right|} \rightarrow \psi \text { and } A_{\rho}\left(x_{n} ; u_{0}\right) \rightarrow\left\|u_{0}\right\|_{\infty}
$$

Definition 3.1. Let $u_{0}$ be a bounded continuous function in $\mathbf{R}^{N}$ and set $M:=\left\|u_{0}\right\|_{\infty}$. A direction $\psi \in S^{N-1}$ is said to be a direction of mean convergence of $u_{0}$ (to $M$ ) if condition $\left(A 3_{\psi}\right)$ is satisfied for $\psi$.

Actually, condition $\left(A 3_{\psi}\right)$ can be converted to the equivalent conditions not invoking the weight $\rho$. Namely, there are some equivalent conditions for $\psi$ to be a direction of mean convergence of $u_{0}$ :

There exists a sequence $\left\{x_{n}\right\} \subset \boldsymbol{R}^{N}$ such that

$$
\left|x_{n}\right| \rightarrow \infty, \frac{x_{n}}{\left|x_{n}\right|} \rightarrow \psi \text { and } u_{0}\left(x+x_{n}\right) \rightarrow\left\|u_{0}\right\|_{\infty} \text { a.e. in } \mathbf{R}^{N} \text { as } n \rightarrow \infty \text {. }
$$

There exists a sequence $\left\{x_{n}\right\} \subset \boldsymbol{R}^{N}$ such that, for each $R>0$,

$$
\left|x_{n}\right| \rightarrow \infty, \frac{x_{n}}{\left|x_{n}\right|} \rightarrow \psi \text { and } \frac{1}{\left|B_{R}\right|} \int_{B_{R}\left(x_{n}\right)} u_{0}(x) d x \rightarrow\left\|u_{0}\right\|_{\infty} \text { as } n \rightarrow \infty .
$$

There exists a sequence $\left\{x_{n}\right\} \subset \boldsymbol{R}^{N}$ and a sequence $\left\{R_{n}\right\}\left(R_{n}>1, R_{n} \rightarrow \infty\right.$ as $n \rightarrow \infty$ ) such that

$$
\left|x_{n}\right| \rightarrow \infty, \frac{x_{n}}{\left|x_{n}\right|} \rightarrow \psi \text { and } \inf _{r \in\left[1, R_{n}\right]} \frac{1}{\left|B_{r}\right|} \int_{B_{r}\left(x_{n}\right)} u_{0}(x) d x \rightarrow\left\|u_{0}\right\|_{\infty} \text { as } n \rightarrow \infty \text {. }
$$

Here and hereafter, $B_{R}(a)$ denotes a $N$-dimensional open ball with radius $R>0$ centered at $a \in \mathbf{R}^{N}$ and $B_{R}=B_{R}(0)$. We refer to [14, Appendix B] for the proof of the equivalence.

Theorem 3.2. Assume $(A 1)$ and $(A 2)$. Let $M=\left\|u_{0}\right\|_{\infty}$ and let $\psi \in S^{N-1}$ 
be a direction of mean convergence of $u_{0}$. Then $u$ blows up at minimal blow-up time and $\psi$ is a blow-up direction. Moreover, for each $R>0$,

$$
\lim _{n \rightarrow \infty} \sup _{x \in B_{R}\left(x_{n}\right)}\left|u(x, t)-v_{M}(t)\right|=0 .
$$

The convergence is uniform on every compact subset of interval $\left(0, T_{M}\right)$.

If we assume a certain growth condition on nonlinear term $f$, we are able to show that the blow-up set is empty unless the initial data is constant, and completely characterize blow-up directions (at minimal blow-up time) by behavior of initial data. Moreover, we are able to obtain a necessary and sufficient condition on initial data for a solution to blow up at minimal blow-up time. The following condition is equivalent to the condition (B) in [8]:

There exist constants $\xi_{0}>0$ and $p>1$ such that

$$
\frac{f(\xi)}{\xi^{p}} \text { is nondecreasing for } \xi \geq \xi_{0} .
$$

This condition means that $f$ grows faster more than polynomial growth.

So far, the next condition is the weakest assumption to show these results, although it looks more complicated.

There exist $\Phi \in C^{2}(0, \infty), c>0$ and $\eta_{1} \geq 0$ such that

$$
\begin{aligned}
& \Phi(\eta)>0, \Phi^{\prime}(\eta) \geq 0 \text { and } \Phi^{\prime \prime}(\eta) \geq 0 \text { for } \eta \geq \eta_{1} ; \\
& \int_{1}^{\infty} \frac{d \eta}{\Phi(\eta)}<\infty ; \\
& f^{\prime}(\eta) \Phi(\eta)-f(\eta) \Phi^{\prime}(\eta) \geq c \Phi(\eta) \Phi^{\prime}(\eta) \quad \text { for } \eta \geq \eta_{1} .
\end{aligned}
$$

This kind of condition was originally introduced by Friedman and McLeod [5] to show that, what is called, a single-point blow-up does occur for bell shaped radially decreasing initial data $u_{0}$ under the assumption $\Delta u_{0}+f\left(u_{0}\right) \geq 0$ which guarantees that $u_{t} \geq 0$ and it has been re-formulated into weaker version by Fujita and Chen [4] and Chen [1]. One can also find a quasilinear version of this condition in Mochizuki and Suzuki [11].

Theorem 3.3. Assume $(A 1),(A 2)$ and $(G U)$ (or $(F M)$ ). Let $u$ be a solution of (1.1) with initial data $u_{0}$ which has minimal blow-up time. Then the following hold:

(i) If the initial data $u_{0}$ is not a constant, then the solution $u$ blows up only at space infinity, that is, its blow-up set is empty.

(ii) A direction is a blow-up direction of $u$ if and only if it is a direction of mean convergence of $u_{0}$. 
Combining Corollary 2.4 and Theorem 3.3, we obtain necessary and sufficient conditions for a solution of (1.1) blows up at minimal blow-up time.

Theorem 3.4. Assume $(A 1),(A 2)$ and $(G U)$ (or $(F M)$ ). Suppose that $u_{0} \not \equiv 0$. Then the solution $u$ of (1.1) blows up at minimal blow-up time if and only if the initial data $u_{0}$ has at least one direction of mean convergence.

Corollary 3.5. Assume the same hypotheses with Theorem 3.4. Let $u$ be a solution of (1.1). Then $u$ blows up at minimal blow-up time if and only if one of the following two conditions for initial data $u_{0}$ holds:

There exists a sequence $\left\{x_{n}\right\} \subset \boldsymbol{R}^{N}$ such that

$$
\begin{gathered}
\left|x_{n}\right| \rightarrow \infty \text { and } u_{0}\left(x+x_{n}\right) \rightarrow\left\|u_{0}\right\|_{\infty} \text { a.e. in } \mathbf{R}^{N} \text { as } n \rightarrow \infty ; \\
\sup _{x \in \mathbf{R}^{N}} A_{\rho}\left(x ; u_{0}\right)=\left\|u_{0}\right\|_{\infty} .
\end{gathered}
$$

Remark 3.6. Giga and Umeda [7] first showed two sufficient conditions on initial data for $\psi \in S^{N-1}$ to be a blow-up direction or non-blow-up direction, respectively and proved that every direction satisfies each of the conditions by a supplementary argument. On the other hand, Seki, Suzuki and Umeda [14] established the formulation of Theorem 3.3(ii) via entirely different approach, adopting a regularizing argument (see Lemma 4.1.2). As above mentioned, their assumption on initial data is equivalent to that of Giga and Umeda [7]. Thus, one is also able to prove Theorem 3.3(ii) by Giga and Umeda's approach if one uses the regularizing argument.

\section{Ideas of proofs}

The proof of Theorem 2.3. The assertion is clear if $u_{0}(x) \equiv M=\left\|u_{0}\right\|_{\infty}$. Thus we may assume that $u_{0}(x) \not \equiv M$. Let $u$ be a solution of (1.1) with minimal blow-up time $T_{M}$. Contrary to the conclusion, assume that there were $t_{1} \in\left[0, T_{M}\right)$ for which

$$
\lim _{R \rightarrow \infty} \sup _{|x| \geq R} u\left(x, t_{1}\right)<v_{M}\left(t_{1}\right) .
$$

If $t_{1} \neq 0$, then we see $L:=\sup _{x \in \mathbf{R}^{N}} u\left(x, t_{1}\right)<v_{M}\left(t_{1}\right)$ in view of (4.1) and the strong maximum principle $([12])$. A comparison argument gives $u(x, t) \leq v_{L}\left(t-t_{1}\right)$ in $\left[t_{1}, t_{1}+T_{L}\right)$, where $v_{L}$ is the solution of $(2.2)$ with initial data $L$ replacing $M$ and $T_{L}=t_{b}(L)$. Then we see $t_{b}\left(u_{0}\right) \geq t_{1}+T_{L}>T_{M}$, which contradicts the assumption that $u$ blows up at minimal blow-up time $T_{M}$, that is, $t_{b}\left(u_{0}\right)=T_{M}$.

As for the case $t_{1}=0$, we take a radially symmetric, radially nonincreasing and continuous function $w_{0}(x)$ in $\mathbf{R}^{N}$ satisfying $u_{0}(x) \leq w_{0}(x) \leq M$ in 
$\mathbf{R}^{N}$. Then the solution $w$ of (1.1) with initial data $w_{0}$ is radially symmetric and radially nonincreasing, and blows up at minimal blow-up time $T_{M}$. Then we reach a contradiction in the same way as the case $t_{1}>0$.

The proof of Theorem 3.2. Let $u$ (or $v$ ) be a supersolution (or subsolution) of (1.1) in $\mathbf{R}^{N} \times\left(0, T_{M}\right)$ satisfying $u, v \leq M$ in $\mathbf{R}^{N} \times[0, T)$ for some $M>0$. Denote by $K$ the Lipschitz constant of function $f$ on $[0, M]$. Then there is an increasing function $C_{K, M}(t)$ such that for $0<t<T$,

$$
\int_{\mathbf{R}^{N}}[v(x, t)-u(x, t)]_{+} \rho(x) d x \leq C_{K, M}(t) \int_{\mathbf{R}^{N}}[v(x, 0)-u(x, 0)]_{+} \rho(x) d x
$$

([14, Appendix A $])$. For a sequence $\left\{x_{n}\right\}$ satisfying $\left(A 3_{\psi}\right)$, we set $u_{n}(x, t):=$ $u\left(x+x_{n}, t\right)$ and substitute $u=u_{n}$ together with $v=v_{M}$. Then we have

$\int_{\mathbf{R}^{N}}\left\{v_{M}(t)-u_{n}(x, t)\right\} \rho(x) d x \leq C_{K, M}(t)\left\{M-A_{\rho}\left(x_{n} ; u_{0}\right)\right\} \rightarrow 0 \quad$ as $n \rightarrow \infty$.

On the other hand, for each $\epsilon \in\left(0, T_{M}\right)$, we see that $u(\cdot, \epsilon) \in B C^{1}\left(\mathbf{R}^{N}\right)$, that is, $u(\cdot, \epsilon)$ is bounded and continuous in $\mathbf{R}^{N}$ up to the first derivative. Thus,

$$
\left\{u_{n}\right\} \text { is uniformly bounded and equicontinuous in } B_{R} \times\left[\epsilon, T_{M}-\epsilon\right]
$$

for every $R>0$. Therefore, we are able to extract a subsequence $\left\{u_{n^{\prime}}\right\}$ such that $\left\{u_{n^{\prime}}\right\}$ converges locally uniformly to some continuous function $w$ by virtue of Ascoli-Arzela theorem. Consequently, we obtain

$$
\int_{B_{R}}\left|v_{M}(t)-w(x, t)\right| \rho(x) d x=0, \quad t \in\left(0, T_{M}\right) .
$$

Namely, $w=v_{M}$. Since the limit is independent of the choice of a subsequence, we conclude $u_{n} \rightarrow v_{M}$. Thus, (3.1) holds and $\psi$ is a blow-up direction.

Remark 4.0.1. Theorem 3.3 can be proved even for degenerate quasilinear parabolic equations of the form $u_{t}=\Delta \phi(u)+f(u)([14])$. For that case, we employ the results on modulus of continuity due to DiBenedetto [3, Lemma $5.2]$ in order to get equicontinuity of $\left\{u_{n}\right\}$.

We present two different procedure to show Theorem 3.3. It is Theorem 3.3(i) that is basic result in both approaches. The first one is a semilinear version of [14], which comes from the method originally introduced by Friedman and McLeod [5] to show that a single-point blow-up does occur for bell shaped radially decreasing initial data. This is valid for very wide class of nonlinear term $f$, such as $f(u)=(u+1)\{\log (u+1)\}^{\beta}$ with $\beta>2$. 
The second one relies on a certain non-blow-up criterion around a given point. It was established by Giga and Kohn [9, Theorem 2.1] for differential inequality $u_{t}-\Delta u \leq K(1+|u|)^{p},(K>0, p>1)$. Our criterion is its direct extension for equations of (1.1) with general nonlinear term $f$ satisfying a certain growth condition. Although it does not allow so wide class of nonlinear term as in the first one, this approach has superiority that it is applicable even to general semilinear parabolic equation whose linear part has variable coefficients and to vector valued equations.

\subsection{The proof of Theorem 3.3 (Part 1)}

By the strong maximum principle, we have $u(x, t)<v_{M}(t)$ in $\mathbf{R}^{N} \times\left(0, T_{M}\right)$. Take $t_{1} \in\left(0, T_{M}\right)$ and fix it. Let $R$ be a positive number and let $w_{0} \in$ $C^{2}\left(B_{R}\right)$ be a radially symmetric and radially nondecreasing function satisfying $u\left(x, t_{1}\right) \leq w_{0}(x) \leq v_{M}\left(t_{1}\right), w_{0}(x)=v_{M}\left(t_{1}\right)$ for $|x|=R$ and $\Delta w_{0}+$ $f\left(w_{0}\right) \geq 0$ in $B_{R}$. Denote by $w$ the solution to the problem

$$
\left\{\begin{array}{l}
w_{t}=\Delta w+f(w), \quad \text { in } B_{R} \times\left(t_{1}, T_{M}\right), \\
w=v_{M}(t), \quad \text { on } \partial B_{R} \times\left(t_{1}, T_{M}\right), \\
w\left(x, t_{1}\right)=w_{0}(x), \quad \text { in } B_{R} .
\end{array}\right.
$$

Lemma 4.1.1. Assume (A1), $(A 2)$ and condition $(F M)$. Then for any compact subset $\Omega \subset B_{R}$,

$$
\sup _{(x, t) \in \Omega \times\left(\tau, T_{M}\right)} w(x, t)<\infty .
$$

Proof. We only use well-known technique which has been used in the study of blow-up sets since it was developed by [5] to show a single-point blow-up phenomenon. See [14, Proposition 2.6] for detail.

If $A_{\rho}\left(0 ; u\left(t_{1}\right)\right) \leq L$ for some $L<M$, there is a point $z \in \mathbf{R}^{N}$ such that $u\left(z, t_{1}\right) \leq L^{\prime}$ for some $L^{\prime} \in(L, M)$. We consider the solution $w$ to the problem (4.2) as a supersolution of (1.1). In order to construct a function $w_{0}(x)$ as used in (4.2) independently of $z$, we make use of equicontinuity of the solution $u$ or a result on modulus of continuity ([3, Lemma 5.2]). As a result, for any $L^{\prime \prime} \in\left(L^{\prime}, M\right)$ there exists $r_{0}>0$ not depending on $z$ such that $u\left(x, t_{1}\right) \leq L^{\prime \prime}$, for $|x-z|<r_{0}$. Using Lemma 4.1.1 with sufficiently large $R>0$, we are able to show the following lemma:

Lemma 4.1.2. Assume $(A 1),(A 2)$ and condition $(F M)$. If $A_{\rho}\left(0 ; u_{0}\right) \leq L$ for some $L<M:=\left\|u_{0}\right\|_{\infty}$, then there exists a constant $C_{M, L}$ such that

$$
u(0, t) \leq C_{M, L} \quad \text { for } t \in\left(0, T_{M}\right) .
$$

The proof of Theorem 3.3. The statement of Theorem 3.3(i) is immediate 
consequence of Lemma 4.1.1. Indeed, we are able to construct, locally in $\mathbf{R}^{N}$, a supersolultion of (1.1) having no blow-up points, taking $R>0$ large enough. We shall prove (ii) of Theorem 3.3. We have only to show that if $u_{0}$ does not satisfy $\left(A 3_{\psi}\right)$, then $\psi$ cannot be a blow-up direction. Assume that $\left(A 3_{\psi}\right)$ does not hold for some $\psi \in S^{N-1}$. Then there exists an open neighborhood $D$ of $\psi$ such that

$$
\sup _{x /|x| \in D} A_{\rho}\left(x ; u_{0}\right) \leq L<M .
$$

For $z \in \mathbf{R}^{N}$ such that $z /|z| \in D$, set $u_{z}(x, t):=u(x+z, t)$. Application of Lemma 4.1.2 to $u_{z}$ implies

$$
u_{z}(0, t)=u(z, t) \leq C_{M, L} \quad \text { for } t \in\left(0, T_{M}\right) .
$$

Since $C_{M, L}$ is independent of $z$, we see, with the aid of Lemma 4.1.2, that $\psi$ is a non-blow-up direction.

\subsection{The proof of Theorem 3.3 (Part 2)}

We first establish a criterion to see whether a point in $\mathbf{R}^{N}$ is a non-blow-up point. Its prototype is found in Giga and Kohn [9, Theorem 2.1].

Lemma 4.2.1. Assume (A1), $(A 2)$ and condition $(G U)$. Let $p$ be the constant appearing in the condition $(G U)$ and let a be a point in $\boldsymbol{R}^{N}$. Then there exists a constant $\delta_{0} \in(0,1]$ having the following property:

(i) Suppose that $1<p \leq 3$. If for some $\delta \in\left(0, \delta_{0}\right), r>0$ and $\tau \in\left(0, T_{M}\right)$,

$$
u(x, t) \leq \delta v_{M}(t), \quad \text { for }(x, t) \in B_{r}(a) \times\left(\tau, T_{M}\right),
$$

and $u$ solves the equation (1.1) in $B_{r}(a) \times\left(0, T_{M}\right)$, then $u$ is locally bounded around the point $a$ at $t=T_{M}$.

(ii) Suppose that $p>3$. Then the statement of (i) holds true with $\delta_{0}=1$.

Remark 4.2.2. This result is stated in [7, Lemma 3.7]. However, there is a flaw in their proof. Indeed, we have to divide the argument into three cases according to the value of $p$, where $p$ is the constant appearing in the condition $(G U)$. Unfortunately, the argument in [7, Lemma 3.7] works only for the case $1<p<3$. However, the proof is completed by reduction to the case $p \geq 3$. We take the opportunity to correct the proof.

The proof of Lemma 4.2.1. Translating the coordinates in space variables and by scaling, we may assume, without loss of generality, that $a=0$ and $r=1$. In the proof we denote by $C$ a generic positive constant possibly changing from line to line. Consider a cutoff function $\phi \in C_{0}^{\infty}\left(B_{1}\right)$ satisfying 
$0 \leq \phi \leq 1$ and $\phi \equiv 1$ on $B_{1 / 2}$. The function $w(t)=w(x, t):=\phi(x) u(x, t)$ satisfies equation

$$
w_{t}=\Delta w-g(x, t)+\phi(x) f(u), \quad \text { in } B_{1} \times\left(0, T_{M}\right),
$$

where $g(x, t)=2 \nabla \cdot(u \nabla \phi)-u \Delta \phi$. Then the representation formula of solutions gives us

$$
w(t)=e^{(t-\tau) \Delta} w(\tau)+\int_{\tau}^{t} e^{(t-s) \Delta}(-g(s)+\phi f(u(s))) d s,
$$

where $\left\{e^{t \Delta}\right\}_{t \geq 0}$ is the heat semigroup in $B_{1}$ with Dirichlet boundary condition. Using the $L^{\infty}-L^{\infty}$ estimates, we have

$$
\left\|e^{(t-s) \Delta} g(s)\right\|_{L^{\infty}\left(B_{1}\right)} \leq C(t-s)^{-1 / 2}\|u(s)\|_{L^{\infty}\left(B_{1}\right)} .
$$

Thus we obtain

$$
\begin{gathered}
\|w(t)\|_{L^{\infty}\left(B_{1}\right)} \leq\|w(\tau)\|_{L^{\infty}\left(B_{1}\right)}+\int_{\tau}^{t} C(t-s)^{-1 / 2}\|u(s)\|_{L^{\infty}\left(B_{1}\right)} d s \\
+\int_{\tau}^{t}\|w(s)\|_{L^{\infty}\left(B_{1}\right)}\left\|\frac{f(u(s))}{u(s)}\right\|_{L^{\infty}\left(B_{1}\right)} d s .
\end{gathered}
$$

(In [7], estimating the integrand of the last term of (4.4) as (4.5) below, they use the Gronwall type inequality ([9, Lemma 2.3$])$. However, since the integrand of the middle term of (4.4) has a singularity, the assumption of the lemma does not satisfied.)

By condition $(G U)$ and (2.2), it is easily seen that $v_{M}(t) \leq C\left(T_{M}-\right.$ $t)^{-1 /(p-1)}$. There are three cases to consider according to the value of $p$. The first case is $p>3$. The integrand of the second term is bounded by $C(t-s)^{-1 / 2}\left(T_{M}-s\right)^{-1 /(p-1)}$, so that its integral with respect to $s$ over interval $[\tau, t]$ is bounded by a constant when $p>3$. Since $\xi \mapsto f(\xi) / \xi$ is nondecreasing by condition $(G U)$, it follows from assumption (4.3) that

$$
\int_{\tau}^{t}\left\|\frac{f(u(s))}{u(s)}\right\|_{L^{\infty}\left(B_{1}\right)} d s \leq \delta^{p-1} \int_{\tau}^{t} \frac{f\left(v_{M}(s)\right)}{v_{M}(s)} d s .
$$

Therefore usual Gronwall's inequality implies

$$
\|w(t)\|_{L^{\infty}\left(B_{1}\right)} \leq C \exp \left(\int_{\tau}^{t}\left\|\frac{f(u(s))}{u(s)}\right\|_{L^{\infty}\left(B_{1}\right)} d s\right) \leq C v_{M}(t)^{\delta^{p-1}} .
$$

Hence we obtain

$$
u(x, t) \leq C v_{M}(t)^{\delta} \quad \text { in } B_{1 / 2} \times\left(\tau, T_{M}\right) .
$$

since $\phi \equiv 1$ on $B_{1 / 2}$. We repeat this manipulation again together with $\phi \in C_{0}^{\infty}\left(B_{1 / 2}\right)$ satisfying $0 \leq \phi \leq 1$ and $\phi \equiv 1$ on $B_{1 / 4}$. Then we obtain

$$
u(x, t) \leq C+\exp \left(\int_{\tau}^{t}\left\|\frac{f(u(s))}{u(s)}\right\|_{L^{\infty}\left(B_{1 / 2}\right)} d s\right) \text { in } B_{1 / 4} \times\left(\tau, T_{M}\right) .
$$


On the other hand, it follows from condition $(G U)$ that

$$
f(\eta \xi) \leq \eta^{p} f(\xi) \quad \text { for } 0<\eta<1, \xi \geq \eta^{-1} \xi_{0} .
$$

From (4.6) and (4.7), we see

$$
\begin{aligned}
& \int_{\tau}^{t}\left\|\frac{f(u(s))}{u(s)}\right\|_{L^{\infty}\left(B_{1 / 2}\right)} d s \leq \int_{\tau}^{t} \frac{f\left(C v_{M}(s)^{\delta}\right)}{v_{M}(s)^{\delta}} d s \\
& \leq C \int_{\tau}^{t} v_{M}(s)^{p(\delta-1)-\delta} f\left(v_{M}(s)\right) d s=C \int_{v_{M}(\tau)}^{v_{M}(t)} v^{p(\delta-1)-\delta} d v \leq C(M, p, \delta, \tau) .
\end{aligned}
$$

Thus we have proved a local boundedness of $u ; u(x, t) \leq C$ in $B_{1 / 4} \times\left(\tau, T_{M}\right)$.

The proof of the local boundedness is almost the same for the case $p=3$ although it is more involved.

We shall consider the case $1<p<3$. This case is more difficult to prove than the case $p \geq 3$. We shall return to the estimate (4.4). In this case, an elementary calculation yields

$$
\begin{aligned}
\int_{\tau}^{t}(t-s)^{-1 / 2}\|u(s)\|_{L^{\infty}\left(B_{1}\right)} d s & \leq C(N, p) \delta\left(T_{M}-t\right)^{1 / 2-1 /(p-1)} \\
& \leq C(N, p) \delta \int_{\tau}^{t}\left(T_{M}-\sigma\right)^{-1 / 2-1 /(p-1)} d \sigma
\end{aligned}
$$

(see [9, Lemma 2.2] for detail). We write $\tilde{\delta}=\delta^{p-1}$ for simplicity of notation. Using the Gronwall type inequality ([9, Lemma 2.3]), we obtain

$$
\begin{aligned}
& \|w(t)\|_{L^{\infty}\left(B_{1}\right)} \\
& \leq\left[\|w(\tau)\|_{L^{\infty}\left(B_{1}\right)}+C(N, p) \delta \int_{\tau}^{t}\left(T_{M}-\sigma\right)^{-1 / 2-1 /(p-1)}\right. \\
& \left.\times \exp \left(-\tilde{\delta} \int_{\tau}^{\sigma} \frac{f(v(\xi))}{v(\xi)} d \xi\right) d \sigma\right] \exp \left\{\tilde{\delta} \int_{\tau}^{t} \frac{f(v(s))}{v(s)} d s\right\} \\
& \leq C(M, N, p, \tau) \delta\left[v(\tau)+\int_{\tau}^{t}\left(T_{M}-\sigma\right)^{-1 / 2-(1-\tilde{\delta}) /(p-1)} d \sigma\right]\left(T_{M}-t\right)^{-\tilde{\delta} /(p-1)} \\
& \leq C(M, N, p, \tau)\left(T_{M}-t\right)^{1 / 2-1 /(p-1)}
\end{aligned}
$$

provided that $\delta$ is chosen so that $1 / 2-(1-\tilde{\delta}) /(p-1)<0$. It follows that

$$
u(x, t) \leq C(M, N, p, \tau)\left(T_{M}-t\right)^{1 / 2-1 /(p-1)} \text { in } B_{1 / 2} \times\left(\tau, T_{M}\right) .
$$

We iterate this argument finitely many times. Take the smallest integer $k$ such that $k>1 /(p-1)$. After $k$ steps later, we have

$$
u(x, t) \leq C_{k}(M, N, p, \tau)\left(T_{M}-t\right)^{-1 / 2(p-1)} \quad \text { in } B_{2^{-k}} \times\left(\tau, T_{M}\right),
$$

and then $u(x, t) \leq C_{k+1}(M, N, p, \tau)\left(T_{M}-t\right)^{-\tilde{\delta} /(p-1)}$ in $B_{2^{-k-1}} \times\left(\tau, T_{M}\right)$. We argue similarly to the case $p \geq 3$, so that we get a bound; $u(x, t) \leq C$ 
in $B_{2^{-k-2}} \times\left(\tau, T_{M}\right)$.

When $u_{0} \not \equiv\left\|u_{0}\right\|_{\infty}$, one is able to prove the existence of $\delta$ as in the condition (4.3). The next lemma is reorganization of [7, Lemma 3.1, Lemma 3.4].

Lemma 4.2.3. Assume $(A 1)$ and $(A 2)$. Suppose that $u_{0} \not \equiv\left\|u_{0}\right\|_{\infty}$. Then for any $\epsilon>0$, there exist $\eta \in(0,1)$ and $\tau \in\left(0, T_{M}\right)$ such that

$$
u(x, t) \leq \epsilon v(t) \quad \text { in } B_{\eta} \times\left(\tau, T_{M}\right) .
$$

Proof. Let $\hat{u}$ be a solution to the linear problem

$$
\left\{\begin{array}{l}
\hat{u}_{t}=\Delta \hat{u}, \quad \text { in } B_{1} \times\left(0, T_{M}\right), \\
\hat{u}=u(x, t), \quad \text { on } \partial B_{1} \times\left(0, T_{M}\right), \\
\hat{u}(x, 0)=u_{0}(x), \quad \text { in } B_{1} .
\end{array}\right.
$$

Then it is not difficult to show that the assertion (4.8) for $\hat{u}$ (see [7, lemma $3.2]$ for detail). Let $t_{1} \in\left(0, T_{M}\right)$. We claim that there exists $\delta \in(0,1)$ such that

$$
u(x, t) \leq \delta v_{M}(t) \quad \text { in } B_{1} \times\left(t_{1}, T_{M}\right) .
$$

Let $w$ be the solution of the heat equation $w_{t}=\Delta w$ in $\mathbf{R}^{N} \times(0, \infty)$ with initial data $w(x, 0)=M^{-1} u_{0}(x)$. Applying the strong maximum principle yields $w<1$ in $\mathbf{R}^{N} \times(0, \infty)$, so that there is $\delta \in(0,1)$ such that $w \leq \delta$ in $B_{1} \times\left(t_{1}, T_{M}\right]$. We set $\bar{u}:=v_{M} w$ and observe that it is a supersolution to (1.1). Thus we see $u \leq \bar{u} \leq \delta v_{M}$ in $B_{1} \times\left(t_{1}, T_{M}\right)$ and the claim (4.9) follows.

Let $G(x, y, t, s)$ be a fundamental solution of the heat equation for the Dirichlet problem in $B_{1}$. In view of positivity of $G$, there is a constant $c>0$ such that $c \leq \int_{B_{1}} G(x, y, t, s) d y \leq 1$ for $x \in B_{\eta}$. Then if we choose $t$ sufficiently close to $T_{M}$, we have

$$
\begin{aligned}
u-\hat{u} & =\int_{0}^{t} \int_{B_{1}} G(x, y, t, s) f(u(y, s)) d y d s \\
& \leq \int_{0}^{t_{1}} f(v(s)) d s \int_{B_{1}} G(x, y, t, s) d y+\int_{t_{1}}^{t} f(\delta v(s)) d s \int_{B_{1}} G(x, y, t, s) d y \\
& \leq\left(\delta^{p}+\epsilon\right) \int_{t_{1}}^{t} f(v(s)) d s \int_{B_{1}} G(x, y, t, s) d y \leq\left(\delta^{p}+\epsilon\right) v(t) \quad \text { for } x \in B_{\eta} .
\end{aligned}
$$

Hence, choosing $\epsilon$ so small as to satisfy $\delta^{p}+2 \epsilon<\delta$, we get a better estimate: $u(x, t) \leq\left(\delta^{p}+2 \epsilon\right) v(t)$ in $B_{\eta} \times\left(t_{1}, T_{M}\right)$. Iterating this manipulation finitely many times yields the assertion (4.8).

The proof of Theorem 3.3. We shall prove Theorem 3.3(i). Let $a$ be a point in $\mathbf{R}^{N}$. Take $R>0$ sufficiently large so that $a \in B_{R}$. It is proved that there exists $\delta \in(0,1)$ such that

$$
u(x, t) \leq \delta v_{M}(t) \quad \text { in } B_{R} \times\left(0, T_{M}\right) .
$$


in the same way to derive (4.9). Actually, it holds with any $\epsilon>0$ replacing $\delta$ by Lemma 4.2.3. Therefore, we see that the solution $u$ is locally bounded around the point $a$ at $t=T_{M}$ via Lemma 4.2.1.

Note that if $u\left(z, t_{1}\right) \leq L^{\prime}<M$ for some $t_{1} \in\left[0, T_{M}\right), L<L^{\prime}<M$ and $z \in \mathbf{R}^{N}$, one is able to construct supersolution $w$ independently of $z$ as in Part 1. The constant $\delta \in(0,1)$ in (4.10) exsits independently of $z$. Then the statement (ii) is obtained similarly to that of Part 1 since one is able to show Lemma 4.1.2 under the assumptions $(A 1),(A 2)$ and $(G U)$ by using Lemma 4.2.1 and Lemma 4.2.3 instead of Lemma 4.1.1.

\section{Some examples}

We shall demonstrate some examples of directional blow-up. Let $u$ be a solution to the Cauchy problem (1.1) which blows up at minimal blowup time. We denote by $S\left(u_{0}\right)\left(\subset S^{N-1}\right)$ the set of all blow-up directions. It is a closed set in $S^{N-1}$. Indeed, for any $\varphi \in S^{N-1} \backslash S$, there exist a constant $C>0$ and an open neighborhood $D \subset S^{N-1}$ of $\varphi$ such that $\sup _{x /|x| \in D} u(x, t) \leq C$. It follows that there are no blow-up directions in $D$. Thus $S^{N-1} \backslash S\left(u_{0}\right)$ is an open set in $S^{N-1}$.

Example 1. (Single directional blow-up) We give an example of initial datum such that $S\left(u_{0}\right)$ consists of a single direction. Let $\psi \in S^{N-1}$ be a direction and set $D=\{r \psi ; r \geq 0\}$. For a point $x \in \mathbf{R}^{N}$, take a point $p_{x} \in D$ such that $\operatorname{dist}\{x, D\}=\left|x-p_{x}\right|$. Let $P$ be a paraboloid defind by $P=\left\{x \in \mathbf{R}^{N} ;\left|x-p_{x}\right| \leq \sqrt{\left|p_{x}\right|}\right\}$ and let $Q=\left\{x \in \mathbf{R}^{N} ;\left|x-p_{x}\right| \leq 2 \sqrt{\left|p_{x}\right|}\right\}$. We choose an initial datum $u_{0} \in B C\left(\mathbf{R}^{N}\right)$ so as to fulfill

$$
\left\{\begin{array}{l}
u_{0}(x)=M \text { if } x \in P,|x| \geq 1 ; \\
0 \leq u_{0}(x)<M \text { if } x \in Q \backslash P ; \\
u_{0}(x)=0 \text { if } x \notin Q .
\end{array}\right.
$$

Let $u$ be a solution of (1.1) with initial datum $u_{0}$. Take a sequence $x_{n}=n \psi$ $(n=1,2, \ldots)$. Clearly, the sequence $\left\{x_{n}\right\}$ satisfies condition $\left(A 3_{\psi}\right)$, so that $\psi$ is a direction of mean convergence of $u_{0}$. Thanks to Theorem 3.3, it is a blow-up direction of $u$. Let $\varphi \neq \psi$ be another direction. Then there are an open neighborfood $G$ of $\varphi$ in $S^{N-1}$ and a constant $r_{0}>0$ such that $\left\{x \in \mathbf{R}^{N} ;|x| \geq r_{0}, x /|x| \in G\right\} \cap Q=\emptyset$. It follows that

$$
\sup \left\{u_{0}(x) ;|x| \geq r_{0}, x /|x| \in G\right\} \leq L<M
$$

for some $L \in(0, M)$. Consequently, $\varphi$ cannot be a direction of mean convergence of $u_{0}$ to $M$. From Theorem 3.3, we observe that this $\varphi$ is not a blow-up direction of $u$. Thus we obtain $S\left(u_{0}\right)=\{\psi\}$. 


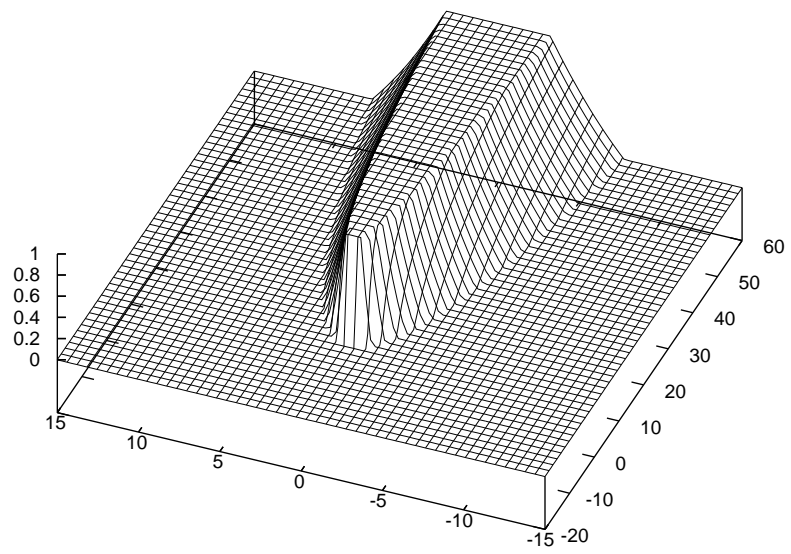

Example 2. (Directional blow-up for an arbitrary given closed set in $S^{N-1}$ ) Let $\tilde{S}$ be a closed subset in $S^{N-1}$. We shall construct an initial datum $u_{0}$ such that $S\left(u_{0}\right)$ coincides with $\tilde{S}$. We set

$$
D=\cup_{r \geq 0} r \tilde{S}=\cup_{r \geq 0}\left\{x \in \mathbf{R}^{N} ; x=r \psi, \psi \in \tilde{S}\right\} .
$$

For a point $x \in \mathbf{R}^{N}$, denote by $p_{x} \in D$ a point in $D$ such that the distance of $x$ and $D$ is achieved; $\operatorname{dist}\{x, D\}=\left|x-p_{x}\right|$. Let $P=\cup\left\{x \in \mathbf{R}^{N} ;\left|x-p_{x}\right| \leq\right.$ $\left.\sqrt{\left|p_{x}\right|}\right\}$ and let $Q=\cup\left\{x \in \mathbf{R}^{N} ;\left|x-p_{x}\right| \leq 2 \sqrt{\left|p_{x}\right|}\right\}$, where the notation " $\cup$ " runs for all such $p_{x}$. We define an initial datum $u_{0} \in B C\left(\mathbf{R}^{N}\right)$ so as to fulfill

$$
\left\{\begin{array}{l}
u_{0}(x)=M \text { if } x \in P,|x| \geq 1 ; \\
0 \leq u_{0}(x)<M \text { if } x \in Q \backslash P ; \\
u_{0}(x)=0 \text { if } x \notin Q .
\end{array}\right.
$$

Then it is proved by the way similar to Example 1 that $S\left(u_{0}\right)=\tilde{S}$.

Example 3. (No direction of mean convergence of almost periodic initial data) Let us restrict ourselves to one-dimensional problem. It is easily see that if the initial data is a non-constant periodic function, then it has no direction of mean convergence, so that the corresponding solution does not blow up at minimal blow-up time. In fact, this is also true for almost periodic functions. Here a function $F$ defined for $-\infty<x<\infty$ is called almost periodic, if for any $\epsilon>0$ there exists a trigonometric polynomial $T_{\epsilon}(x)$ such that

$$
\left|F(x)-T_{\epsilon}(x)\right|<\epsilon, \quad-\infty<x<+\infty .
$$

Proof. Let $u_{0}$ be an almost periodic function in $\mathbf{R}$ which is not a constant and let $M$ be its maximum. Contrary to the conclusion, suppose that $u_{0}$ 
had a direction of mean convergence to $M$. Then there exists a sequence $\left\{x_{n}\right\} \subset \mathbf{R}$ such that $u_{0}\left(x+x_{n}\right) \rightarrow M$ a.e. in $\mathbf{R}$ as $n \rightarrow \infty$. From a characteristic property of almost periodic functions, one can extract a subsequence, which is also denoted by $\left\{x_{n}\right\}$, such that the convergence $u_{0}\left(x+x_{n}\right) \rightarrow M$ is uniform (see [2, Chapter 1]). Take a point $y \in \mathbf{R}$ such that $m:=u_{0}(y)<M$. Define $H:=M-m$ and $z_{n}:=y-x_{n}$. Then $\left|u_{0}\left(z_{n}+x_{n}\right)-M\right|=H$ for all $n$. This means that there is no subsequence $\left\{x_{n^{\prime}}\right\} \subset\left\{x_{n}\right\}$ for which the sequence $u_{0}\left(x+x_{n^{\prime}}\right)$ is uniformly convergent. This is a contradiction.

Acknowledgement. The second author is grateful to Mr. Tsuyoshi Yoneda for his discussion on almost periodic functions in Example 3. The work of the first author was partly supported by the Grant-in-Aid for Scientific Reserch, No.17654037, No.18204011, the Japan Society of the Promotion of Science (JSPS) and by COE "Mathematics of Nonlinear Structures via Singularities" (Hokkaido University) sponsored by JSPS. The works of the second and third authors were supported by the 21st century COE Program "Base for New Development of Mathematics to Science and Technology" sponsored by JSPS at Graduate School of Mathematical Sciences, the University of Tokyo.

\section{References}

[1] Y.-G. Chen, On blow-up solutions of semilinear parabolic equations; Analytical and numerical studies, Thesis(Ph.D.)-University of Tokyo, 1988.

[2] C. Corduneanu, Almost Periodic Functions, Interscince Publishers, New York, 1968.

[3] E. DiBenedetto, Continuity of weak solutions to a general porous medium equation, Indiana Univ. Math. J. 32(1983), 83-118.

[4] H. Fujita and Y.-G. Chen, On the set of blow-up points and asymptotic behaviours of blow-up solutions to a semilinear parabolic equation, Analyse Mathématique et Applications, Contrib. Honneur Jaques-Louis Lions, (1988), 181-201.

[5] A. Friedman and B. McLeod, Blow-up of positive solutions of semilinear heat equations, Indiana Univ. Math. J. 34(1985), 425-447.

[6] Y. Giga and N. Umeda, On blow-up at space infinity for semilinear heat equations, J. Math. Anal. Appl. 316(2006), 538-555.

[7] Y. Giga and N. Umeda, Blow-up directions at space infinity for solutions of semilinear heat equations, Bol. Soc. Paran. Mat. 23(2005), 9-28. 
[8] Y. Giga and N. Umeda, Correction to "Blow-up directions at space infinity for solutions of semilinear heat equations", Bol. Soc. Paran. Mat, to appear.

[9] Y. Giga and R.V. Kohn, Nondegeneracy of blow-up for semilinear heat equations, Comm. Pure Appl. Math. 42(1989) 845-884.

[10] A. A. Lacey, The form of blow-up for nonlinear parabolic equations, Proc. R. Soc. Edinb., Sect. A 98(1984), 183-202.

[11] K. Mochizuki and R. Suzuki, Blow-up sets and asymptotic behavior of interfaces for quasilinear degenerate parabolic equations in $\boldsymbol{R}^{N}$, J. Math. Soc. Japan 44 (1992), 485-504.

[12] M. H. Protter and H. F. Weinberger, Maximum principles in Differential Equations, Englewood Cliffs, N.J. Prentice-Hall, 1967.

[13] Y. Seki, On directional blow-up for quasilinear parabolic equations with fast diffusion, J. Math. Anal. Appl, to appear.

[14] Y. Seki, R. Suzuki and N. Umeda, Blow-up directions for quasilinear parabolic equations, Proc. R. Soc. Edinb., Sect. A, in press. 